Received 00th January 20xx, Accepted 00th January 20xx DOI: $10.1039 / x 0 x \times 00000 x$

\title{
ViscY NMR experiments in phosphoric acid as viscous solvent for the individualization of small molecules within mixtures by spin diffusion
}

\begin{abstract}
Francois Pedinielli, ${ }^{a}$ Ritchy Leroy, ${ }^{a}$ Agathe Martinez, ${ }^{a}$ Jean-Marc Nuzillard ${ }^{a}$ and Pedro Lameiras*a
The analysis of small molecules within complex mixtures is a particularly difficult task when dealing with the study of metabolite mixtures or chemical reaction media. This issue has fostered in recent years an active search for effective and practical solutions. In this context, the ViscY NMR approach was recently proposed. ViscY collectively designates the NMR experiments that take advantage of spin diffusion in highly viscous solvents or solvent blends for the individualization of the NMR spectra of small molecule mixture components. Two viscous media were prepared from ortho-phosphoric acid $(85 \%)$ solution by dilution with either $\mathrm{D}_{2} \mathrm{O}$ or DMSO- $d_{6}$, thus providing solvent blends with slightly different polarities in which all liquid-state NMR experiments can be carried out easily. Two mixtures, one of four structurally close dipeptides and one of four low-polarity phosphorus-containing compounds were used for method assessment, using ViscY experiments such as homonuclear selective $1 \mathrm{D}$ and $2 \mathrm{D}{ }^{1} \mathrm{H}$ NOESY experiments, heteronuclear $2 \mathrm{D}{ }^{1} \mathrm{H}-{ }^{15} \mathrm{~N} /{ }^{1} \mathrm{H}-{ }^{31} \mathrm{P}$ HSQCNOESY and ${ }^{1} \mathrm{H}-{ }^{13} \mathrm{C} /{ }^{1} \mathrm{H}-{ }^{15} \mathrm{~N} /{ }^{1} \mathrm{H}^{-31} \mathrm{P} \quad \mathrm{NOAH}$ experiments.
\end{abstract}

\section{Introduction}

Liquid-state NMR spectroscopy plays a key role in the structure elucidation of unknown organic compounds, provided that sample purity is fair to excellent. Real-life situations may preclude the resort to purification methods and force to analyse mixtures. For instance, the substances produced by chemical synthesis often contain starting materials, by-products, and the desired end product. As well, natural or biotechnological extracts may contain several hundred organic molecules. In this context, simultaneous purification and NMR spectroscopy through LC-SPE-NMR or LC-SPEMS/NMR coupling strategies may be carried out. ${ }^{1-4}$ The physical separation of mixture constituents may be helpful but is not always practical. Moreover, chromatography cannot be applied to highly reactive molecules, is unappealing if crucial molecular associations are shattered by separation and may be simply too time-consuming and therefore too expensive to employ. Consequently, using NMR without prior physical separation may turn out to be relevant.

The study of mixtures by liquid-state NMR mainly focuses on the identification and quantification of already known molecules. Determining their structures may be demanding in case of accidental strong peak overlap. Being able to assign each resonance to a specific compound should limit the necessity of chromatographic separation, help scientists analyse unknown compounds within mixture, and thus significantly improve the

\footnotetext{
${ }^{a}$ Université de Reims Champagne-Ardenne, CNRS, ICMR UMR 7312, 51097 Reims, France. E-mail: pedro.lameiras@univ-reims.fr, Tel:+33 326918228.

$+\quad$ Electronic Supplementary Information (ESI) available. See DOI: $10.1039 / \mathrm{x} 0 \times x \times 0000 \mathrm{x}$
}

efficiency of synthetic and natural product chemistry. So far, NMR spectroscopy has been considered for mixture analysis in a reduced number of ways: i) The translational diffusion coefficient in solution $(D)$ is a molecular property and describes its mobility. The individual 1D NMR spectrum of each mixture component can be extracted by considering $D$ as discriminating factor, using the 2D DOSY method. ${ }^{5-}$ ${ }^{8}$ Nonetheless, $D$ values are only weakly discriminating, even if experimental techniques may improve their resolving power in special cases (adjunction of a chromatographic solid phase, interaction of the molecules of interest with soluble polymers, lanthanide shift reagents, or micelles). 9-18 ii) Multi-quantum spectroscopy coupled (or not) with broadband homonuclear decoupling / pure shift NMR, ultrafast data acquisition, sparse sampling, and tensor decomposition methods may all help separate the spectra of molecules within mixtures. ${ }^{19-23}$ iii) The use of viscous solvents under appropriate conditions slows down the tumbling rate of small and mid-sized molecules in solution, ${ }^{24-36}$ so that the longitudinal cross-relaxation regime induces spin diffusion over entire molecular spin networks. Therefore, the resonances of different species may be distinguished according to their ability to share magnetization through intramolecular spin diffusion. All ${ }^{1} \mathrm{H}$ resonances from each molecule may correlate together in a $2 \mathrm{D}$ NOESY spectrum, making possible to individualize the ${ }^{1} \mathrm{H} N M R$ spectrum of each mixture constituent. In 2008, Simpson and coworkers first published the use of a high-viscosity solvent, the chlorotrifluoroethylene polymer, for organic mixture analysis by means of $1 D$ and 2D NOESY spectroscopy. ${ }^{29}$ The same team reported in 2012 the use of supercooled water in narrow capillaries to manipulate the rotational dynamics of small molecules for identifying mixed small metabolites. ${ }^{31}$ Lameiras et al. published original results in mixture analysis by NMR using glycerol and 
glycerol carbonate (in 2011), ${ }^{30}$ DMSO/glycerol (in 2016), ${ }^{32}$ DMSO/water (in 2017), ${ }^{33}$ sucrose solution and agarose gel (in 2019), ${ }^{34}$ and sulfolane-based solvents (in 2020) ${ }^{36}$ as viscous solvents for ${ }^{1} \mathrm{H}$ and ${ }^{19} \mathrm{~F}$ spin diffusion promotion. In 2020, Lameiras and co-workers introduced the term ViscY for NMR methods in which Viscous solvents promote the mixture component individualization by NMR spin diffusion spectroscopY. ${ }^{36}$

To date, the investigation of high- and low-polarity molecules within mixtures has been only investigated in a few viscous solvents. In this context, two viscous media were prepared from ortho-phosphoric acid ( $85 \%$ ) by dilution with either $\mathrm{D}_{2} \mathrm{O}$ or DMSO$d_{6}$, thus providing solvent blends with slightly different polarities. For method assessment, two mixtures, one of four structurally close dipeptides and one of four low-polarity phosphorus-containing compounds were studied by ViscY experiments, including homonuclear selective $1 \mathrm{D}$ and $2 \mathrm{D}{ }^{1} \mathrm{H}$ NOESY experiments, heteronuclear 2D ${ }^{1} \mathrm{H}-{ }^{15} \mathrm{~N} /{ }^{1} \mathrm{H}^{31} \mathrm{P}$ HSQC-NOESY and ${ }^{1} \mathrm{H}^{13} \mathrm{C} /{ }^{1} \mathrm{H}-$ ${ }^{15} \mathrm{~N} /{ }^{1} \mathrm{H}-{ }^{31} \mathrm{P}$ NOAH experiments.

Orthophosphoric acid $\mathrm{H}_{3} \mathrm{PO}_{4}$, also named phosphoric acid, is a weak mineral triacid. It plays an important role in inorganic chemistry and biochemistry. About $90 \%$ of its production is engaged in the industry of fertilizers. At room temperature, pure phosphoric acid is a crystalline solid. It is usually sold as a colourless syrup of $85 \%$ concentration in water. This liquid is miscible with polar solvents such as alcohol, water, and most non-polar solvents. ${ }^{37}$ Phosphoric acid (85\%) solution presents a melting point around 299 K. ${ }^{38}$ Its melting point can be reduced by the addition of more water or of DMSO- $d_{6}$. By the way, melting points of phosphoric acid (85\%) solution respectively reached $248 \mathrm{~K}$ and $258 \mathrm{~K}$ after adding $20 \%$ of water and $30 \%$ of DMSO- $d_{6}$ by volume. This property enabled us to prepare liquid-state NMR samples at or below room temperature, which is particularly suitable for molecules of poor thermal stability. As a result, spin diffusion may occur on a wide temperature range, from room to negative Celsius temperatures owing to the viscosity increase of both solvent blends upon temperature decrease. ${ }^{39}$ However, the viscosity of the phosphoric acid-based blends at room temperature stays relatively low, even though phosphoric acid $(85$ $\%$ in water) presents a viscosity of $37.59 \mathrm{cP}$ at $298 \mathrm{~K}^{39}$ (for comparison $\eta_{\text {water }}=0.898 \mathrm{cP}$ and $\eta_{\mathrm{DMSO}}=2.003 \mathrm{cP}$ at $\left.25^{\circ} \mathrm{C}\right)^{40}$ As a consequence, NMR samples tubes can be prepared without any issues, contrary to highly viscous solvents such as glycerol $(\eta=934$ CP at $298 \mathrm{~K}){ }^{41}$ Furthermore, the weak triacidity of phosphoric acid solutions ( $\mathrm{pKa}=2.148,7.198$, and 12.319 at $298 \mathrm{~K}$ ) makes its use compatible with most of small molecules without the risk of altering them. However, considering these phosphoric acid-based solvents forces to suppress the strong ${ }^{1} \mathrm{H}$ NMR signal of water, a requirement that it is easily fulfilled by the excitation sculpting sequence. ${ }^{42}$ In addition, the high amount of added DMSO- $d_{6}$ and $\mathrm{D}_{2} \mathrm{O}$ easily enables automatic field-locking and shimming, like for any other common NMR solvent. Nonetheless, the major disadvantage of using phosphoric acid solutions remains the formation of salts that may disturb the matching of the spectrometer probehead which requires care when handling a cryoprobe. As a result, the duration of pulses may be longer than that measured in usual organic deuterated solvents. Owing to the higher dielectric constant of water compared to DMSO- $d_{6},{ }^{43}$ the phosphoric acid $(85 \%) / \mathrm{D}_{2} \mathrm{O}$ mixture is more suitable for the investigation of high-polarity molecules while the binary phosphoric acid $(85 \%) /$ DMSO- $d_{6}$ mixture is more appropriate for the study of low-polarity compounds. Medium-sized polar and lowly-polar compounds require a low amount of water or DMSO- $d_{6}$ in phosphoric acid (85\%) solutions whereas smaller or more flexible compounds necessitate more water or DMSO- $d_{6}$ for driving spin diffusion from room temperature to negative Celsius temperatures.

The criterion for optimal temperature selection is a compromise between overall spectral resolution and intensity of NOESY crosspeaks between nuclei that are not nearby to reveal a NOE signal that would be absent in a low-viscosity solution. A temperature reduction enhances spin diffusion but also reduces peak height through line broadening due to a more active transverse relaxation. Sample cooling is therefore required if the NOESY spectrum shows positive NOE responses (diagonal and off-diagonal peaks of opposite signs). Following the complexity of the mixtures, the analysis of ${ }^{1} \mathrm{H}$ NMR spectra may become intractable owing to ${ }^{1} \mathrm{H}$ resonance overlapping. A conventional remedy to this involves the spreading of the spectroscopic information along a second dimension that encodes chemical shifts of nuclei other than protons. ${ }^{32-34,36}$ This approach to NMR mixture analysis is illustrated in phosphoric acid $(85 \%) / \mathrm{D}_{2} \mathrm{O}$ solution using $2 \mathrm{D}^{1} \mathrm{H}-{ }^{15} \mathrm{~N}$ HSQCNOESY and ${ }^{1} \mathrm{H}^{13} \mathrm{C} /{ }^{1} \mathrm{H}^{15} \mathrm{~N}$ NOAH experiments and in phosphoric acid (85\%)/DMSO- $d_{6}$ solution using $2 \mathrm{D}{ }^{1} \mathrm{H}-{ }^{31} \mathrm{P}$ HSQC-NOESY and ${ }^{1} \mathrm{H}_{-}{ }^{31} \mathrm{P}$ NOAH experiments offering ${ }^{1} \mathrm{H} /{ }^{13} \mathrm{C} /{ }^{15} \mathrm{~N}$ and ${ }^{1} \mathrm{H} /{ }^{31} \mathrm{P}$ chemical shifts of respectively every mixed high- and low-polarity molecules.

\section{Results and discussion}

\section{Leu-Val, Leu-Tyr, Gly-Tyr and Ala-Tyr mixture in phosphoric acid} $(85 \%) / D_{2} \mathrm{O}(8: 2, v / v)$ solution.

These dipeptides when dissolved in pure water are not discriminated according to their translational diffusion behaviour, owing to their comparable molecular weight and shape (Fig. S1 in the Electronic Supplementary Information (ESI) file). In this context, we have considered the resolving power of homo- and heteronuclear NOESY-based spin diffusion experiments in viscous phosphoric acid solutions to offer a working alternative to the DOSY experiment.

An experimental issue of handling phosphoric acid solution is the mandatory suppression of its strong residual ${ }^{1} \mathrm{H}$ water signal (see Fig. S2a) to fully benefit from the high dynamic range of the NMR spectrometer receiver since deuterated phosphoric acid solution is quite expensive and the chemical exchange between solvent deuterium nuclei and dipeptide amide protons may happen. Nonetheless, the suppression of the HOD signal is easily achieved by using an excitation sculpting sequence (Fig. S2b). ${ }^{42}$

Since temperature is a crucial parameter to observe spin diffusion because it is related to solvent viscosity and therefore to overall rotational correlation times $\tau_{C},{ }^{30,44}$ we have determined the optimal temperature at which NOE cross-peaks were positive (negative NOE enhancements, slow motion regime), well-resolved, and as intense as possible between nuclei (not close enough to display a NOE in low viscosity solvent). The optimized temperature of $288 \mathrm{~K}$ has been chosen by recording NOESY experiments with water suppression by excitation sculpting (see Fig. 1a, Fig. S3: amide 
proton region NOESY spectra at 298, 288 and $278 \mathrm{~K}$ and S4: full NOESY spectrum at $288 \mathrm{~K}$ in ESI).

The use of an aqueous phosphoric acid solution enables a thorough intramolecular magnetization transfer through spin diffusion, observed over distances of $>14 \AA$ within each small and flexible dipeptide in the NOESY spectrum at $288 \mathrm{~K}$ whereas in water at $298 \mathrm{~K}$ the NOESY spectrum reveals fewer NOESY cross-peaks, and of opposite sign (positive NOE enhancements, fast motion regime, see Fig. 1b, full NOESY spectrum in Fig. S5 of ESI). As a result, under Visc $Y$ conditions, the compound by compound clustering of proton resonances is achieved, making possible the individualization of each mixture component since the chemical shift pattern of each dipeptide is predictable. The individualization of the four dipeptides in water would have necessitated the concomitant use of J-coupling and dipolar-coupling ${ }^{1} \mathrm{H}$ NMR experiments such as COSY/TOCSY and NOESY, following the usual peptide resonance assignment method.

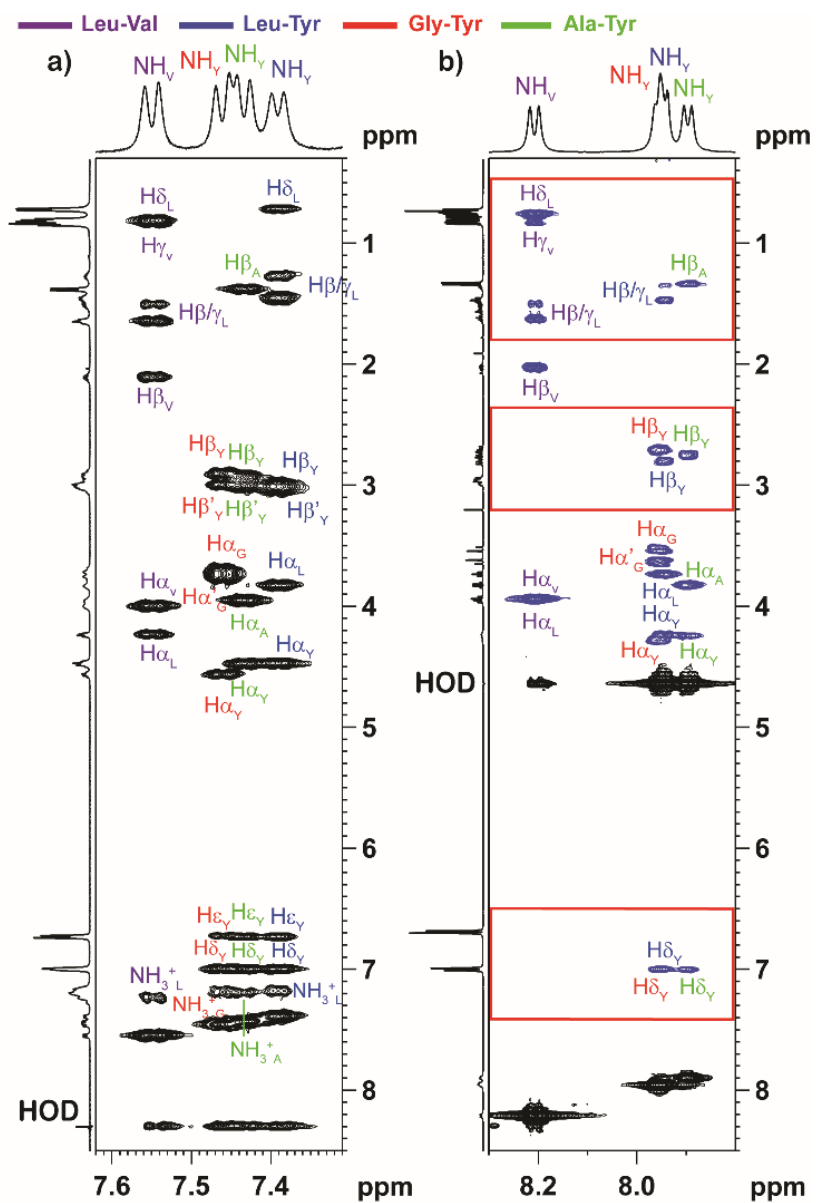

Fig. 1 Amide proton region of 2D NOESY spectra of the dipeptide test mixture $(20 \mathrm{mM})$, mixing time $\left(t_{m}\right)=1 \mathrm{~s}$, at $500 \mathrm{MHz}\left({ }^{1} \mathrm{H}\right)$, dissolved a) in phosphoric acid $(85 \%) / \mathrm{D}_{2} \mathrm{O}(8: 2, \mathrm{v} / \mathrm{v})$ solution, at 288 $\mathrm{K}$, b) dissolved in $\mathrm{H}_{2} \mathrm{O} / \mathrm{D}_{2} \mathrm{O}(9: 1, \mathrm{v} / \mathrm{v})$, at $298 \mathrm{~K}$. The red frames correspond to spectral regions of interest in which water as solvent has a major effect on the number and sign of observable NOESY cross-peaks.
For complex mixture investigations, detecting only the resonances of interest during signal acquisition may deliver additional structural information by preventing proton resonance overlapping. Selectively exciting a suitable set of proton resonances in 1D selective NOESY experiments exemplifies this approach.

In the 1D selective NOESY experiment, a single spin is excited, its magnetization is further flipped to bring it to the $z$-axis where it can spread by spin diffusion along with the molecular proton network. The 1D selective NOESY pulse sequence starts with a multiplet selective excitation block (Fig. 2e). ${ }^{46,47}$ Two wideband inversion pulses have been included during the mixing time. Their position has been adjusted to circumvent the resurgence of the strong water signal during the acquisition time that comes from longitudinal relaxation. $^{47}$

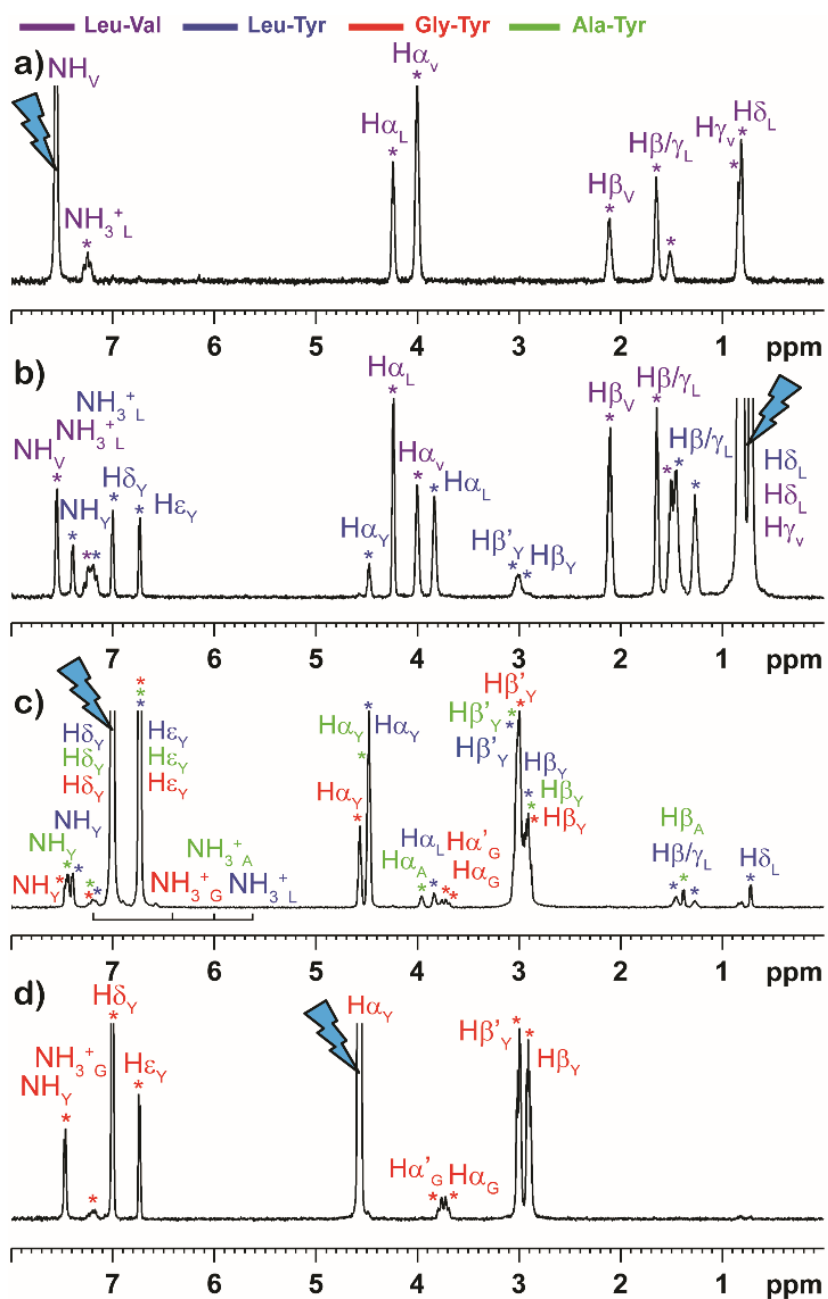

e)

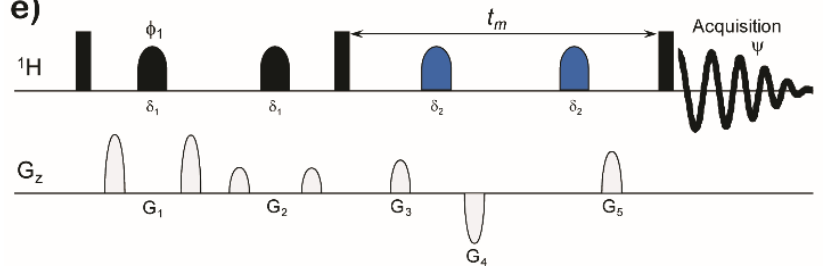

Fig. 2 Multiplet selective excitation 1D ${ }^{1} \mathrm{H}$ NOESY spectra of the dipeptide test mixture $(20 \mathrm{mM})$ dissolved in phosphoric acid $(85 \%) / D_{2} \mathrm{O}(8: 2, \mathrm{v} / \mathrm{v})$ solution $(\mathrm{a}, \mathrm{b}, \mathrm{c}, \mathrm{d}, 288 \mathrm{~K}), t_{m}=1 \mathrm{~s}$, at $500 \mathrm{MHz}$ $\left({ }^{1} \mathrm{H}\right)$. The initial selective inversion pulses excite: a) the $\mathrm{NH}_{\mathrm{V}}(\mathrm{LV})$ 
proton resonance; b) the $\mathrm{H} \delta_{\mathrm{L}}(\mathrm{LY}) / \mathrm{H} \delta_{\mathrm{L}}(\mathrm{LV}) / \mathrm{H} \gamma_{\mathrm{V}}(\mathrm{LV})$ proton resonances; $\mathrm{c})$ the $\mathrm{H} \delta_{\mathrm{Y}}(\mathrm{LY}) / \mathrm{H} \delta_{\mathrm{Y}}(\mathrm{GY}) / \mathrm{H} \delta_{\mathrm{Y}}(\mathrm{AY})$ proton resonances; $\left.\mathrm{d}\right)$ the $H \alpha_{Y}(G Y)$ proton resonance. e) Pulse sequence: $\varphi=x, y,-x,-y, \psi$ $=x,-x$.

The 1D selective NOESY spectra in Fig. 2 show that each dipeptide is discriminated by spin diffusion in phosphoric acid $(85 \%) / D_{2} \mathrm{O}(8: 2$, $\mathrm{v} / \mathrm{v}$ ) solution at $288 \mathrm{~K}$, using a suitable set of selectively excited proton resonances. By the way, the selective excitation of the $\mathrm{NH}$ amide proton at $7.55 \mathrm{ppm}$ discloses a magnetization exchange solely with the protons of the Leu-Val dipeptide, even with terminal ammonium protons $\mathrm{NH}_{3}{ }^{+}$of leucine (Fig. 2a) due to the acidity of the medium allowing to sufficiently slow down the chemical exchange with water. The selective excitation of the side chain $\mathrm{H} \delta$ and $\mathrm{H} \gamma$ protons (between 0.65 and $0.95 \mathrm{ppm}$ ) reveals a magnetization exchange with all protons of the two Leu-Val and Leu-Tyr dipeptides (even with terminal ammonium protons $\mathrm{NH}_{3}{ }^{+}$of both leucines) (Fig. 2b). By comparison with the 1D NOESY spectra in Fig. 2a and 2b, a full proton assignment of Leu-Tyr is achievable. The selective excitation of the aromatic $\mathrm{H} \delta$ protons of Leu-Tyr, GlyTyr and Ala-Tyr presents all proton resonances of Leu-Tyr, Gly-Tyr and Ala-Tyr (including terminal ammonium protons $\mathrm{NH}_{3}{ }^{+}$of alanine and glycine) (Fig. 2c). The complete individual ${ }^{1} \mathrm{H}$ spectrum of GlyTyr is obtained after selectively exciting the $\mathrm{H} \alpha$ proton at $4.57 \mathrm{ppm}$ (Fig. 2d).

Another way to discriminate each mixed dipeptide, by detecting only resonances of interest during signal acquisition, has been to resort to only one selective NOESY experiment namely a $F_{1}$ band selective $F_{1}$ decoupled 2D NOESY experiment ${ }^{48,49}$. Amide $\mathrm{NH}$ resonances have been the initial source of magnetization after the initial band selective excitation step since their resonances are close in frequency and they are not scalarly coupled together (Fig. 3). The mixing time block, including wideband inversion pulses to avoid the reappearance of the HOD signal, let spin diffusion extend throughout the proton network of each dipeptide. Carrying out this latter experiment in phosphoric acid solution allows the assignment of all proton resonances of Leu-Val, Leu-Tyr, Gly-Tyr and Ala-Tyr under Visc $Y$ conditions.

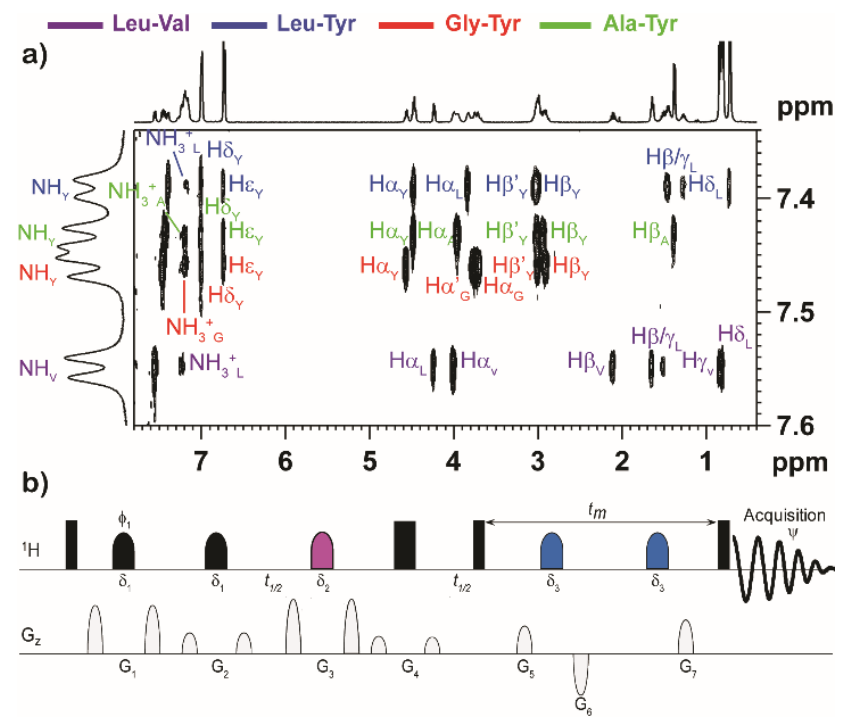

Fig. 3 a) $F_{1}$ band selective $F_{1}$ decoupled $2 \mathrm{D}{ }^{1} \mathrm{H}$ NOESY spectrum of the dipeptide test mixture $(20 \mathrm{mM})$ in phosphoric acid $(85 \%) / \mathrm{D}_{2} \mathrm{O}$
$(8: 2, v / \mathrm{v})$ solution, at $288 \mathrm{~K}$, at $500 \mathrm{MHz}\left({ }^{1} \mathrm{H}\right), t_{m}=1 \mathrm{~s}$. b) Pulse sequence: $\varphi_{1}=x, y,-x,-y, \psi=x,-x$. The initial selective $180^{\circ}$ pulses had a Gaussian shape and were applied to the four $\mathrm{NH}$ amide proton resonances.

It may happen in more complex mixtures that components of interest do not reveal resolved proton resonances due to too severe spectral overlap. In such cases, the larger chemical shift dispersion of ${ }^{15} \mathrm{~N}$ nuclei may turn out to be useful. By coupling the HSQC and NOESY experiments, an entire proton spectrum should be recorded for a molecule, starting only from a single nitrogen resonance. Along this way, the $2 \mathrm{D}{ }^{1} \mathrm{H}^{15}{ }^{15} \mathrm{~N}$ HSQC-NOESY spectrum of the dipeptide test mixture, recorded at $288 \mathrm{~K}$, in phosphoric acid $(85 \%) / \mathrm{D}_{2} \mathrm{O}(8: 2, \mathrm{v} / \mathrm{v})$ solution unveils that all protons of each dipeptide within mixture correlate with all other protons (except all terminal ammonium protons $\mathrm{NH}_{3}{ }^{+}$for a sensitivity issue) after having marked the nitrogen chemical shift in $F_{1}$ under Visc $Y$ conditions (Fig. 4).

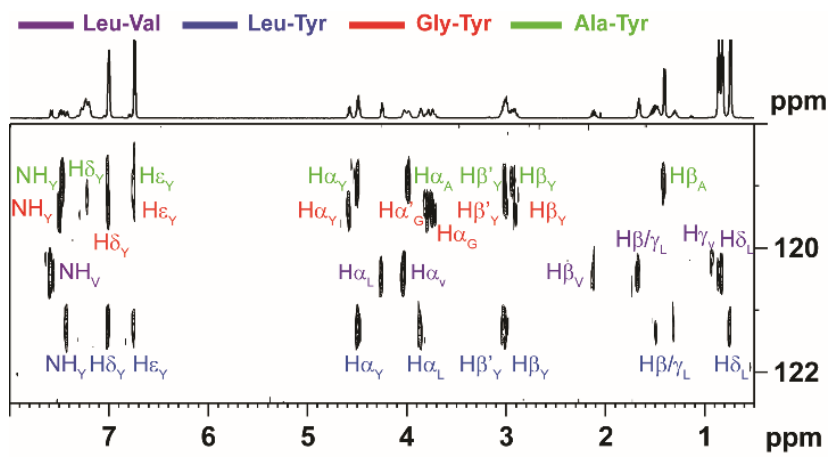

Fig. $42 \mathrm{D}{ }^{1} \mathrm{H}-{ }^{15} \mathrm{~N}$ HSQC-NOESY spectrum of the dipeptide test mixture $(50 \mathrm{mM})$ dissolved in phosphoric acid $(85 \%) / \mathrm{D}_{2} \mathrm{O}(8: 2, \mathrm{v} / \mathrm{v})$ solution, $t_{m}=1 \mathrm{~s}$, at $288 \mathrm{~K}$, at $600 \mathrm{MHz}\left({ }^{1} \mathrm{H}\right)$.

Another alternative way to individualize each dipeptide within mixture has been also to focus on the NOAH-5 BMSCN supersequence allowing in a row, with only one recycling delay, the acquisition of ${ }^{1} \mathrm{H}_{-}{ }^{13} \mathrm{C} \mathrm{HMBC}(\mathrm{B}),{ }^{1} \mathrm{H}_{-}{ }^{15} \mathrm{~N} \mathrm{HMQC}(\mathrm{M}),{ }^{1} \mathrm{H}_{-}{ }^{13} \mathrm{C} \mathrm{HSOC}(\mathrm{S})$, ${ }^{1} \mathrm{H}-{ }^{1} \mathrm{H}$ COSY $(\mathrm{C})$ and ${ }^{1} \mathrm{H}-{ }^{1} \mathrm{H}$ NOESY $(\mathrm{N})$ spectra under Visc $Y$ conditions (Fig. $\mathrm{S} 6$ in ESI). ${ }^{50}$ In addition to the full assignment of every proton resonances of mixed Leu-Val, Leu-Tyr, Gly-Tyr and Ala-Tyr thanks to the active spin diffusion occurring during the mixing time of the NOESY experiment, heteronuclear ${ }^{1} \mathrm{H}-{ }^{13} \mathrm{C}$ and ${ }^{1} \mathrm{H}-{ }^{15} \mathrm{~N} \quad J$-scalar correlations are also obtained (in particular all nitrogen chemical shifts of terminal ammonium groups $\mathrm{NH}_{3}{ }^{+}$). This additional information caught by a single NMR experiment in a shorter time may prove to be relevant in the case of strong ${ }^{1} \mathrm{H}$ resonance overlapping in the study of complex mixtures.

Dicyclohexyl(4-(N,N-dimethylamino)phenyl) Phosphine, Exophenyl Kwon [2.2.1] Bicyclic Phosphine, Allyltriphenylphosphonium Bromide and (Methoxymethyl)triphenylphosphonium Chloride mixture in phosphoric acid (85\%)/DMSO- $d_{6}(7: 3, v / v)$ solution.

The simplification of small molecule mixture analysis has been extended to ${ }^{31} \mathrm{P}$ NMR spectroscopy using heteronuclear chemical shift resonance labelling. Phosphorus is common in synthetic intermediates and biological compounds. It has a $100 \%$ natural abundance and presents sharp NMR peaks. ${ }^{51}$ Its chemical shift 
range covers about $2000 \mathrm{ppm}$, which facilitates the individualization of phosphorus-containing compounds in mixtures. ${ }^{52}$ In the slow tumbling limit, unlike ${ }^{19} \mathrm{~F}$ and ${ }^{1} \mathrm{H}$ nuclei that reveal negative NOEs and HOEs, ${ }^{31} \mathrm{P}$ nuclei show only positive HOEs that tend towards zero with slower tumbling owing to the particular value of its magnetogyric ratio. ${ }^{53}$. A workaround to this experimental issue is to consider ${ }^{31} \mathrm{P}$ nuclei as chemical shift markers and to induce magnetization exchange along the intramolecular proton network using the 2D ${ }^{1} \mathrm{H}^{31} \mathrm{P}$ HSQC-NOESY experiment under ViscY conditions. We report here the individualization of four mixed phosphorus-containing compounds dissolved in the phosphoric acid $(85 \%) / D M S O-d_{6} \quad(7: 3, v / v)$ solvent blend under spin diffusion conditions by homonuclear selective $1 \mathrm{D}$ and $2 \mathrm{D}{ }^{1} \mathrm{H}$ NOESY experiments, heteronuclear 2D ${ }^{1} \mathrm{H}-{ }^{31} \mathrm{P}$ HSQC-NOESY and ${ }^{1} \mathrm{H}^{31} \mathrm{P}$ NOAH experiments.

Like the dipeptides dissolved in pure water, the four phosphoruscontaining molecules (Fig. 5) do not present any differentiation by translational diffusion in pure DMSO- $d_{6}$, owing to their similar molecular weight (Fig. S7 in ESI). This observation led us to assess phosphoric acid (85\%) mixed with DMSO- $d_{6}$ in the individualization of these four low-polarity molecules by taking profit from the resolving power of NOESY-based spin diffusion experiments. Proton NMR in phosphoric acid $(85 \%) / D M S O-d_{6}(7: 3, v / v)$ solution forces the elimination of its strong residual $\mathrm{H}_{2} \mathrm{O}$ signal. This water signal suppression has been easily accomplished using the excitation sculpting sequence (Fig. S8b in ESI). ${ }^{42}$



Fig. 5 Chemical structures of the four phosphorus-containing compounds within mixture, 1a: dicyclohexyl (4- $(N, \quad N-$ dimethylamino)phenyl) phosphine, 1b: exophenyl Kwon [2.2.1] bicyclic phosphine, 1c: allyltriphenylphosphonium bromide and 1d: (methoxymethyl)triphenylphosphonium chloride.

The effect of temperature on intramolecular spin diffusion has been already discussed in the preceding section. Likewise, we have established the optimal temperature that gives the best compromise between efficient ${ }^{1} \mathrm{H}$ spin diffusion and spectral resolution by using 2D ${ }^{1} \mathrm{H}$ NOESY experiments with water suppression by excitation sculpting, at $500 \mathrm{MHz}\left({ }^{1} \mathrm{H}\right)$, at 308,298 , and $288 \mathrm{~K}$. Spin diffusion is already active at $308 \mathrm{~K}$ for all molecules (Fig. S9 in ESI). Nonetheless, a full magnetization exchange along the proton network of every molecule is visible at $298 \mathrm{~K}$. At $288 \mathrm{~K}$, full spin diffusion is efficient but spectral resolution is a bit damaged due to more active $T_{2}$ transverse relaxation, responsible for ${ }^{1} \mathrm{H}$ resonance line broadening (Fig. S9 in ESI). As a result, the optimal temperature of $298 \mathrm{~K}$ has been chosen for the following NMR studies because it offered efficient ${ }^{1} \mathrm{H}$ spin diffusion (negative NOE enhancements, positive NOE cross-peaks, slow tumbling regime) and suitable ${ }^{1} \mathrm{H}$ spectral resolution.

The 2D NOESY spectrum at $298 \mathrm{~K}$ discloses four positive NOE cross-peak patterns, each corresponding to a single mixture component (Fig. $6 \mathrm{a}$ and Fig. S10 in ESI). The pattern identification of $1 \mathrm{a}$ is driven by the distinctive aromatic protons $\mathrm{H}_{15,19}$ at $7.67 \mathrm{ppm}$ (Fig. 6a, purple pattern). The pattern of $1 \mathrm{~b}$ is identified by means of the tropanic ring $\mathrm{H}_{3}$ at $4.29 \mathrm{ppm}$ (Fig. 6a, blue pattern). The proton $\mathrm{H}_{9}$ at $5.36 \mathrm{ppm}$ helps distinguish the proton pattern of 1c (Fig. 6a, red pattern). The proton resonance $\mathrm{H}_{10}$ at $3.21 \mathrm{ppm}$ is a relevant starting point for recognizing the complete resonance pattern of $1 \mathrm{~d}$ (Fig. 6a, green pattern). As a result, the use of phosphoric acid $(85 \%) / D M S O-d_{6}$ binary solvent enables an entire intramolecular magnetization exchange within every mixed phosphorus-containing compound by spin diffusion, observed over distances greater than $13 \AA$, in part due to the rigidity of the cycloalkyl and aromatic parts. In contrast, when the conventional 2D NOESY spectrum is acquired in pure DMSO- $d_{6}$ at $298 \mathrm{~K}$, the four mixed phosphorus-containing compounds quickly reorient and reveal negative NOE cross-peaks (positive NOE enhancements, fast motion regime) thus avoiding spin diffusion to be detected (Fig. $6 \mathrm{~b}$ and Fig. S11 in ESI). 


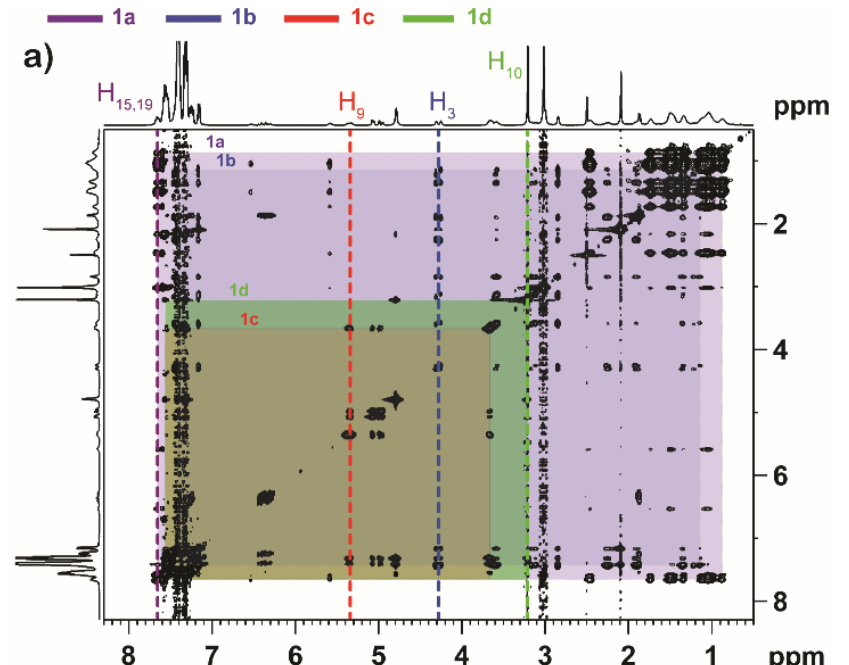

b)

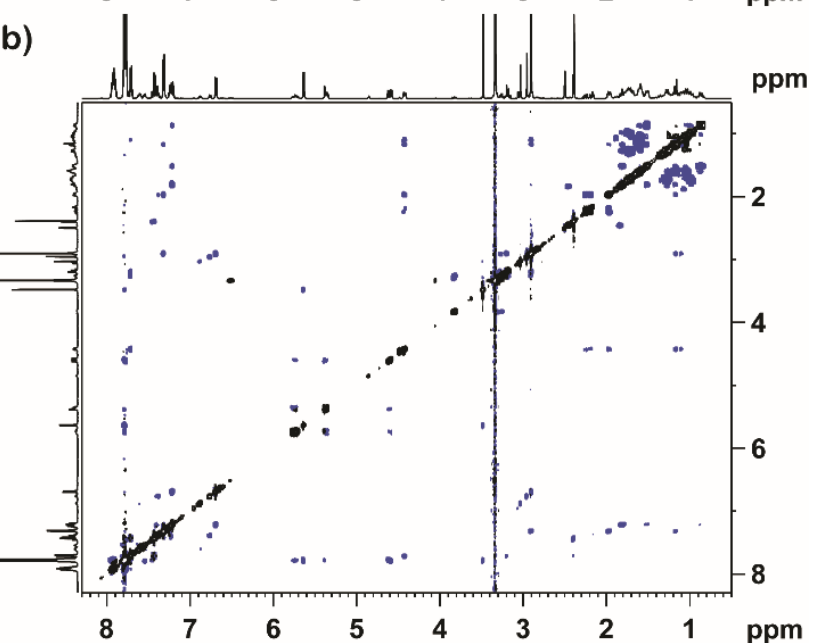

Fig. $6 \quad 2 \mathrm{D} \quad{ }^{1} \mathrm{H}$ NOESY spectra of the phosphorus-containing compound test mixture $(20 \mathrm{mM})$, mixing time $\left(t_{m}\right)=0.5 \mathrm{~s}$, at $298 \mathrm{~K}$, at $500 \mathrm{MHz}\left({ }^{1} \mathrm{H}\right)$, a) dissolved in phosphoric acid (85\%)/DMSO- $d_{6}$ $(7: 3, v / v)$ solution, b) dissolved in neat DMSO- $d_{6}$.

It is not easy to unambiguously assign each proton resonance to each specific compound within mixture due to resonance peak overlaps, particularly in the proton $1 \mathrm{D}$ and $2 \mathrm{D}$ spectra from 0.6 to 2.6 and from 7.1 to $7.8 \mathrm{ppm}$. The multiplet selective excitation 1D NOESY experiment, ${ }^{46,47}$ formerly described in the context of dipeptide mixture analysis, addresses this pitfall by clustering proton resonances belonging to the same molecule. The resurgence of the strong ${ }^{1} \mathrm{H}$ water signal during the mixing time of the NOESY block is still prevented by wideband inversion pulses enclosed in gradient pulse pairs. ${ }^{47}$ Choosing an appropriate set of selectively excited proton resonances enables the acquisition of the individual 1D ${ }^{1} \mathrm{H}$ spectrum of every mixed compound by taking benefit from spin diffusion under ViscY conditions (Fig. 7). In particular, the individualization of $1 \mathrm{a}$ is achieved by the selective excitation of the aromatic protons $\mathrm{H}_{15,19}$ at $7.67 \mathrm{ppm}$ (Fig. 7a). All proton resonances of $1 \mathrm{~b}$ are detected by means of the selective excitation of the tropanic ring proton $\mathrm{H}_{3}$ at $4.29 \mathrm{ppm}$. By the way, spin diffusion is sufficiently active to correlate proton signals from the two aromatic groups (Fig. 7b). Selective excitations of the ethylene protons $\mathrm{H}_{9}$ at
$5.36 \mathrm{ppm}$ and the methyl group $\mathrm{H}_{10}$ at $3.21 \mathrm{ppm}$ respectively group together all proton resonances of $1 \mathrm{c}$ and $1 \mathrm{~d}$ (Fig. $7 \mathrm{c}$ and d).

The selective excitation of resolved proton resonances has permitted to individualize each mixture component by taking profit from spin diffusion under ViscY conditions. Nonetheless, in the investigation of more complex phosphorus-containing mixtures, molecules of interest may not reveal isolated proton resonances due to severe ${ }^{1} \mathrm{H}$ spectral overlap, thus preventing the use of selective $1 \mathrm{D}$ and $2 \mathrm{D}{ }^{1} \mathrm{H}$ NOESY experiments. A remedy may be to take advantage of the broad chemical shift range of ${ }^{31} \mathrm{P}$. By coupling 2D HSQC and NOESY experiments, a complete proton spectrum should be obtained for each component within mixture starting only from one single phosphorus resonance in the indirect dimension. ${ }^{29,32-34,36}$ The 2D ${ }^{1} \mathrm{H}^{-31} \mathrm{P}$ HSQC-NOESY spectrum of the phosphorus-containing compound test mixture dissolved in phosphoric acid (85\%)/DMSO- $d_{6}(7: 3, v / v)$ solution, recorded at 298 $\mathrm{K}$, shows that all protons of each molecule can correlate with all other protons by spin diffusion after having marked the phosphorous chemical shift in $F_{1}$ (Fig. 8). As a result, the capability to individualize phosphorous-containing compounds within mixture is achievable by using the $2 \mathrm{D}^{1} \mathrm{H}-{ }^{31} \mathrm{P}$ HSQC-NOESY experiment under Visc $Y$ conditions, even if CSSF $^{54}$ or GEMSTONE ${ }^{55}$ experiments may be a pertinent alternative to reach the required resolution in traditional NMR solvents.

Another substitute way in the individualization of each mixed phosphorus-containing molecule has been also to concentrate on the NOAH-4 BSCN supersequence to acquire, consecutively, with only one recycling delay, ${ }^{1} \mathrm{H}-{ }^{31} \mathrm{P} \mathrm{HMBC}(\mathrm{B}),{ }^{1} \mathrm{H}-{ }^{31} \mathrm{P} \mathrm{HSQC}(\mathrm{S}),{ }^{1} \mathrm{H}-{ }^{1} \mathrm{H}$ COSY (C) and ${ }^{1} \mathrm{H}-{ }^{1} \mathrm{H}$ NOESY (N) spectra under ViscY conditions (Fig. 9). ${ }^{50}$ The full assignment of the proton resonances of each mixed compound is reachable by taking profit from the active spin diffusion acting during the mixing time of the NOESY experiment, even though the signal/noise ratio is somewhat reduced compared to that of the NOESY spectrum acquired alone due to signal loss by transverse relaxation. Furthermore, multiple bond $J$-scalar ${ }^{1} \mathrm{H}-{ }^{1} \mathrm{H}$ and ${ }^{1} \mathrm{H}^{31} \mathrm{P}$ correlations are additionally observed allowing access to all ${ }^{1} \mathrm{H}$ and ${ }^{31} \mathrm{P}$ chemical shifts. This supplementary information trapped in a single NMR experiment, in complement of the HSQCNOESY experiment, in a reduced time may turn out to be significant in the case of strong ${ }^{1} \mathrm{H}$ resonance overlapping in the study of complex mixtures of unknown molecules. 


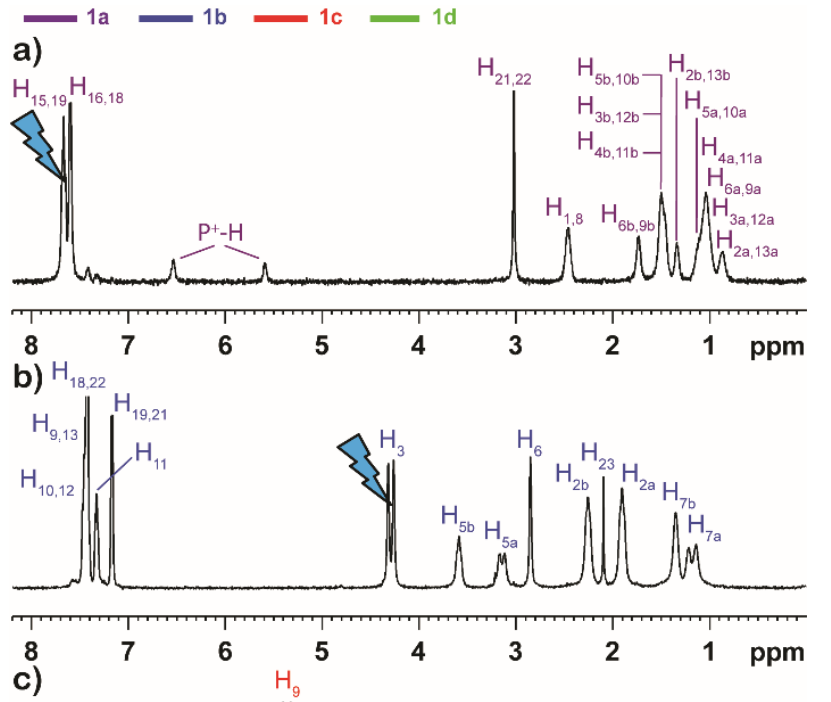

c)

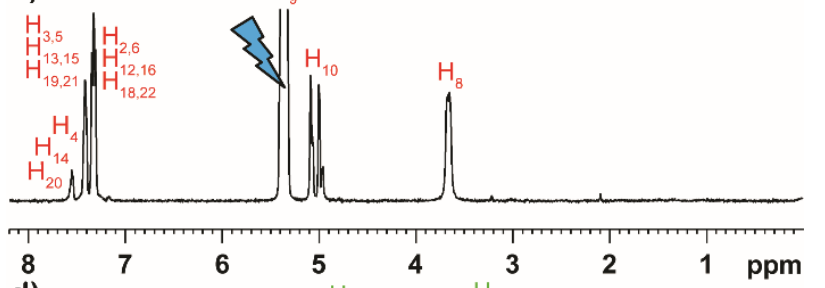

d)

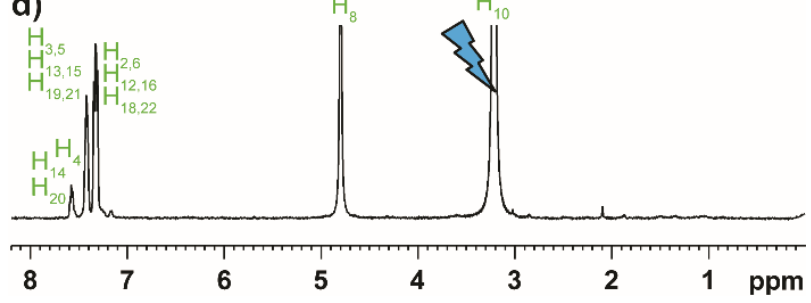

Fig. 7 Multiplet selective excitation 1D ${ }^{1} \mathrm{H}$ NOESY spectra of the phosphorus-containing compound test mixture $(20 \mathrm{mM})$ dissolved in phosphoric acid (85\%)/DMSO- $d_{6}(7: 3, v / v)$ solution $(a, b, c$ and $d)$, $t_{m}=0.5 \mathrm{~s}$, at $298 \mathrm{~K}$, at $500 \mathrm{MHz}\left({ }^{1} \mathrm{H}\right)$. The initial selective inversion pulses excite: a) $H_{15,19}(1 a)$, b) $H_{3}(1 b)$, c) $H_{9}(1 c)$ and d) $H_{10}(1 d$ ) proton resonances.

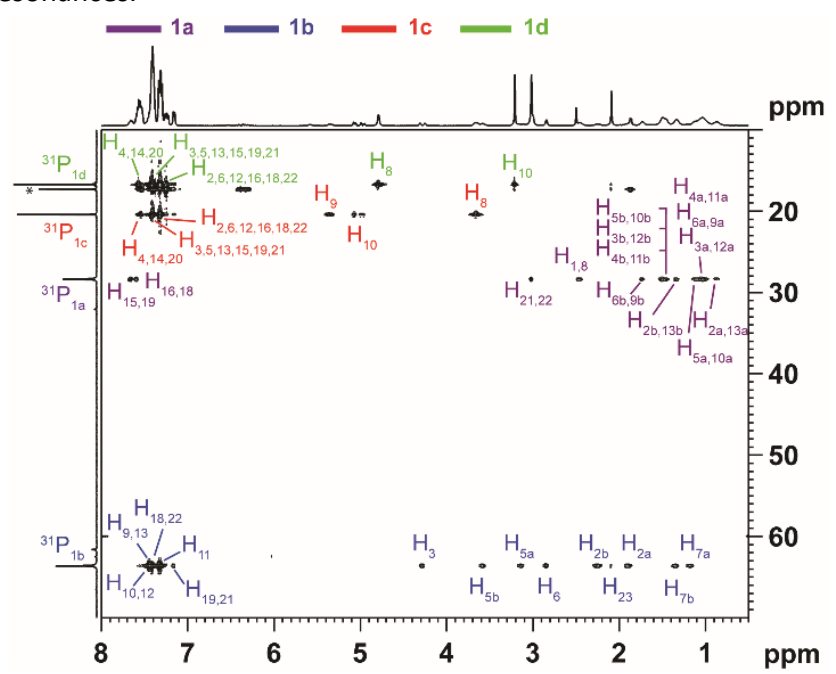

Fig. 8 a) $2 \mathrm{D}{ }^{1} \mathrm{H}-{ }^{31} \mathrm{P}$ HSQC-NOESY spectrum of the phosphoruscontaining compound test mixture $(20 \mathrm{mM})$ dissolved in phosphoric acid $(85 \%) /$ DMSO- $_{6}(7: 3, \mathrm{v} / \mathrm{v})$ solution, $t_{m}=0.5 \mathrm{~s}$, at $298 \mathrm{~K}$, at 500 $\mathrm{MHz}\left({ }^{1} \mathrm{H}\right) .{ }^{*}$ Impurity. 


\section{ARTICLE}

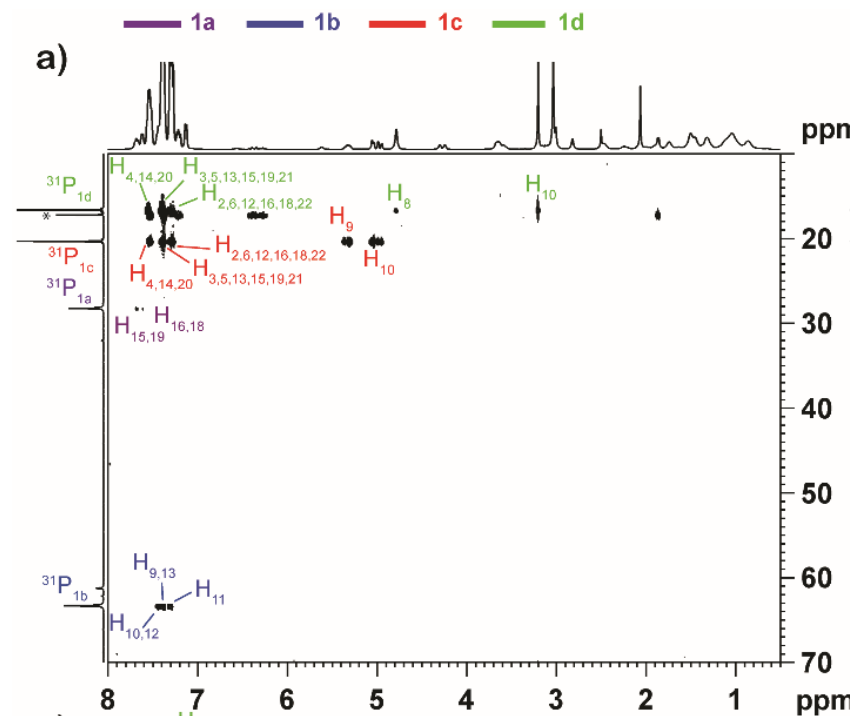

b)

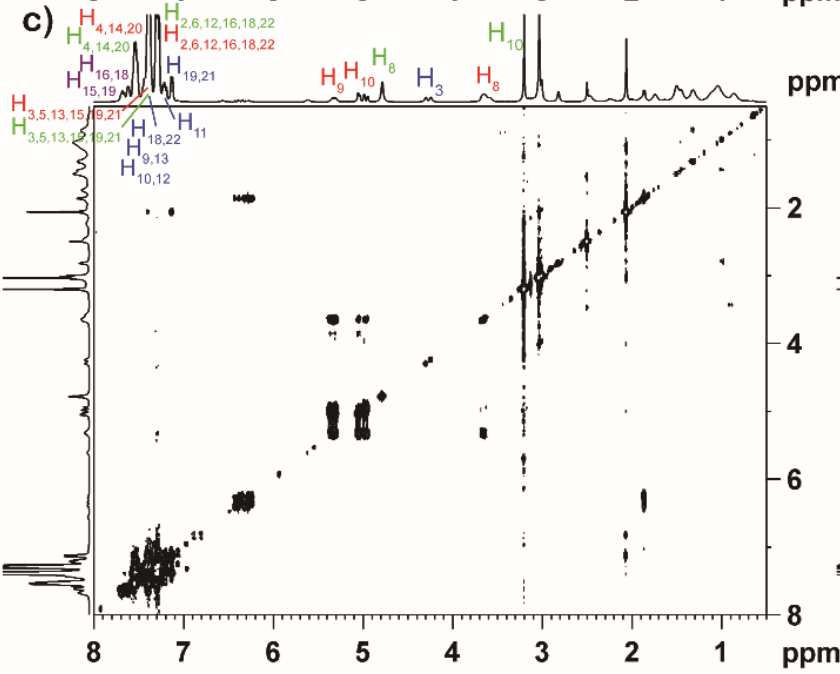

d)

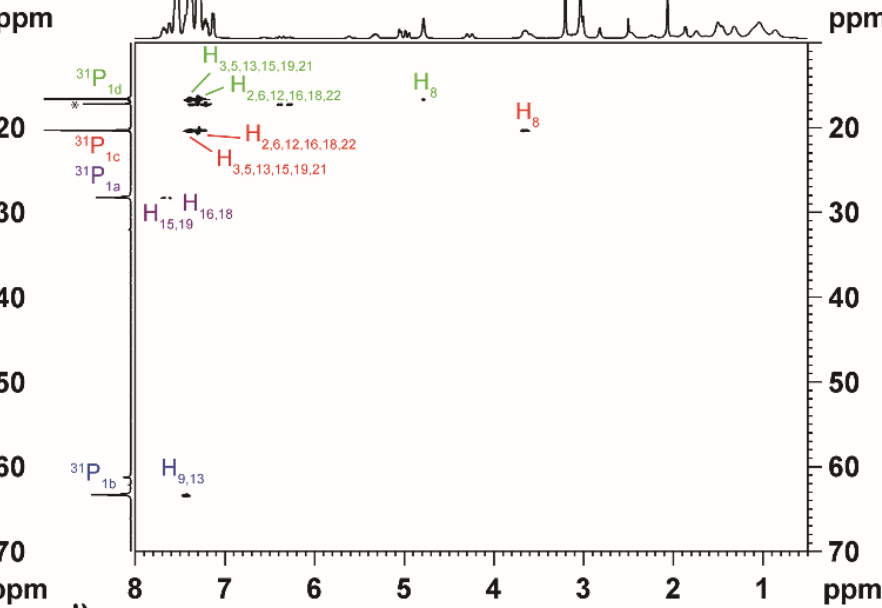

ppm

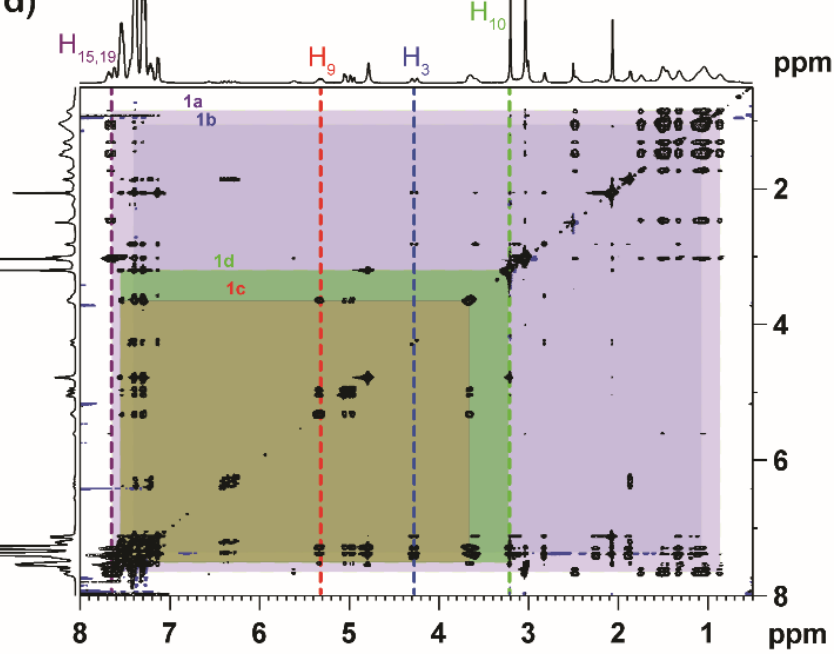

Fig. 9 a) $2 \mathrm{D}{ }^{1} \mathrm{H}-{ }^{31} \mathrm{P} H M B C$, b) ${ }^{1} \mathrm{H}-{ }^{31} \mathrm{P}$ HSQC, c) ${ }^{1} \mathrm{H}-{ }^{1} \mathrm{H}$ COSY and d) ${ }^{1} \mathrm{H}-{ }^{1} \mathrm{H}$ NOESY $\left(t_{m}=0.5 \mathrm{~s}\right.$ ) spectra using the NOAH-4 BSCN sequence of phosphorus-containing compound test mixture $(50 \mathrm{mM})$ dissolved in phosphoric acid $(85 \%) / D M S O-d_{6}(7: 3, v / v)$ solution, at $298 \mathrm{~K}$, at 500 $\mathrm{MHz}\left({ }^{1} \mathrm{H}\right) .{ }^{*}$ Impurity.

\section{Experimental}

Chemical reagents. DMSO- $d_{6}$ and $\mathrm{D}_{2} \mathrm{O}$ were purchased from Eurisotop (Gif-sur-Yvette, France). Leu-Val, Leu-Tyr, Gly-Tyr and AlaTyr were purchased from TCl Europe (Zwijndrecht, Belgium). Dicyclohexyl(4-( $N, N$-dimethylamino)phenyl)phosphine, exophenyl Kwon [2.2.1] bicyclic phosphine and phosphoric acid (85\%, w/w) were purchased from Sigma-Aldrich (Saint-Quentin-Fallavier, France). Allyltri-phenylphosphonium bromide and (methoxymethyl)triphenylphosphonium chloride were purchased from Acros
Organics (Geel, Belgium). All peptides and phosphorus-containing compounds had $95 \%$ or higher purity and were dissolved at a concentration from 20 to $50 \mathrm{mM}$ respectively in phosphoric acid $(85 \%) / \mathrm{D}_{2} \mathrm{O}(8: 2, \mathrm{v} / \mathrm{v})$ and phosphoric acid $(85 \%) /$ DMSO- $_{6}(7: 3, \mathrm{v} / \mathrm{v})$ solutions without exothermic reactions.

NMR Spectroscopy. NMR experiments on the dipeptide and phosphorus-containing compound test mixtures were carried out on Bruker Avance AVIII500 and AVIII600 NMR spectrometers respectively equipped with a $5 \mathrm{~mm} \mathrm{BBFO+} \mathrm{probe} \mathrm{and} \mathrm{a} 5 \mathrm{~mm} \mathrm{TCl}$ cryoprobe using the TOPSPIN software (Rheinstetten, Germany). 
Gradient pulses (respectively maximum $0.535 \mathrm{Tm}^{-1}$ and $0.613 \mathrm{Tm}^{-1}$ ) were generated by a $10 \mathrm{~A}$ amplifier. Sample temperature was controlled by a Bruker variable temperature (BVT) unit supplied with chilled air produced by a Bruker cooling unit.

All dipeptide mixture spectra were referenced so that the tyrosine $\mathrm{H} \alpha$ proton and $\mathrm{C} \alpha$ carbon resonances respectively appeared at 7.00 and $132.00 \mathrm{ppm}$.

All spectra of phosphorus-containing compounds were referenced so that the residual proton signal of DMSO- $d_{6}$ was observed at 2.50 ppm and the phosphorus signal of $\mathrm{H}_{3} \mathrm{PO}_{4}$ was observed at $0.00 \mathrm{ppm}$. Additional NMR data acquisition and processing parameters for Fig. 1-4 and Fig. 6-9 are reported in the Electronic Supplementary Information file.

\section{Conclusions}

The use of phosphoric acid (85\%) mixed with $\mathrm{D}_{2} \mathrm{O}$ or DMSO- $d_{6}$ as viscous binary solvents has been reported for the first time for the individualization of respectively high- and low-polarity components within complex mixtures, by taking benefit from NMR spin diffusion, under Visc $Y$ conditions, at usual magnetic field strengths.

The component individualization of the Leu-Val, Leu-Tyr, Gly-Tyr and Ala-Tyr mixture and the $\operatorname{dicyclohexyl}(4-(N, N$ dimethylamino)phenyl) phosphine, exophenyl Kwon [2.2.1] bicyclic phosphine, allyltriphenylphosphonium bromide and (methoxymethyl)triphenylphosphonium chloride mixture has been respectively carried out, at $288 \mathrm{~K}$, in phosphoric acid $(85 \%) / \mathrm{D}_{2} \mathrm{O}$ $(8: 2, v / v)$ solution using homonuclear selective $1 \mathrm{D}{ }^{1} \mathrm{H}$ NOESY, selective $2 \mathrm{D}{ }^{1} \mathrm{H}$ NOESY and heteronuclear $2 \mathrm{D}{ }^{1} \mathrm{H}-{ }^{15} \mathrm{~N}$ HSQC-NOESY and ${ }^{1} \mathrm{H}_{-}{ }^{13} \mathrm{C} /{ }^{1} \mathrm{H}_{-}{ }^{15} \mathrm{~N} \mathrm{NOAH}$ experiments and at $298 \mathrm{~K}$ in phosphoric acid $(85 \%) / D M S O-d_{6}$ solution using homonuclear selective $1 \mathrm{D}{ }^{1} \mathrm{H}$ NOESY, selective $2 \mathrm{D}^{1} \mathrm{H}$ NOESY and heteronuclear $2 \mathrm{D}{ }^{1} \mathrm{H}-{ }^{31} \mathrm{P} \mathrm{HSQC}-$ NOESY and ${ }^{1} \mathrm{H}^{31} \mathrm{P}$ NOAH experiments.

Considering viscous phosphoric acid-based solvents reveal significant benefits compared to previously described viscous solvents such as glycerol, glycerol carbonate or agarose gel. ${ }^{30,34}$ The spin diffusion is active over a large temperature range, compatible with heat-sensitive molecules, and the preparation of NMR sample tubes is simple. The viscous phosphoric acid (85\%)/water solution blend is rather appropriate for the investigation of high-polarity molecules whereas phosphoric acid $(85 \%) / D M S O-d_{6}$ solution is rather recommended for the study of low-polarity compounds. Medium-sized high- and low-polarity compounds necessitate a low concentration of water or DMSO- $d_{6}$ in phosphoric acid (85\%) whereas smaller or more flexible compounds demand more water or DMSO- $d_{6}$ in phosphoric acid (85\%) until $50 \%$ $(\mathrm{v} / \mathrm{v})$ for driving spin diffusion from room to negative Celsius temperatures, under ViscY conditions. However, phosphoric acid (85\%) solutions may interact with or alter fragile chemical compounds preventing NMR analysis and they may unsettle the probe matching when using a cryoprobe.

Upcoming studies involving the ViscY approach will deal with the study of other polar and apolar mixtures composed of small-sized molecules for assessing the spin diffusion power in viscous phosphoric acid-based solutions.

\section{Author Contributions}

F. P. and P. L. performed NMR data acquisition and processing. F.P., R. L. and A. M. assisted in NMR data analysis. P. L. conceived the study, analysed NMR data, and wrote the Edge article with the contribution from J.-M. N.

\section{Conflicts of interest}

The authors declare no conflict of interest.

\section{Acknowledgements}

Financial support to the PIAneT CPER project was provided by CNRS, Conseil Régional Champagne Ardenne, Conseil Général de la Marne, Ministry of Higher Education and Research (MESR), and EU-programme FEDER. We kindly thank $\mathrm{Dr} \overline{\mathrm{E}}$. Kupče and $\operatorname{Dr}$ T. D. W. Claridge for having given us the microprograms of the NOAH pulse sequences.

\section{Notes and references}

1. M. Spraul, A. S. Freund, R. E. Nast, R. S. Withers, W. E. Maas and O. Corcoran, Anal. Chem., 2003, 75, 1546-1551.

2. O. Corcoran and M. Spraul, Drug Discov. Today, 2003, 8, 624-631.

3. M. Godejohann, L.-H. Tseng, U. Braumann, J. Fuchser and M. Spraul, J. Chrom. A, 2004, 1058, 191-196.

$4 . \quad$ J.-L. Wolfender, J.-M. Nuzillard, J. J. J. van der Hooft, J.-H. Renault and S. Bertrand, Anal. Chem., 2019, 91, 704-742.

5. K. F. Morris and C. S. Johnson, J. Am. Chem. Soc., 1992, 114, 3139-3141.

6. K. F. Morris and C. S. Johnson, J. Am. Chem. Soc., 1993, 115, 4291-4299.

7. K. F. Morris, P. Stilbs and C. S. Johnson, Anal. Chem., 1994, 66, 211-215.

8. A. A. Colbourne, G. A. Morris and M. Nilsson, J. Am. Chem. Soc., 2011, 133, 7640-7643.

9. S. Viel, F. Ziarelli and S. Caldarelli, Proc. Natl. Acad. Sci. USA, 2003, 100, 9696-9698.

10. G. Pages, C. Delaurent and S. Caldarelli, Angew. Chem. Int. Ed. , 2006, 45, 5950-5953.

11. G. Pages, C. Delaurent and S. Caldarelli, Anal. Chem., 2006, 78, 561-566.

12. S. Caldarelli, Magn. Reson. Chem., 2007, 45, S48-S55.

13. C. Carrara, S. Viel, F. Ziarelli, G. Excoffier, C. Delaurent and S. Caldarelli, J. Magn. Reson., 2008, 194, 303-306.

14. R. Evans, S. Haiber, M. Nilsson and G. A. Morris, Anal. Chem., 2009, 81, 4548-4550.

15. M. E. Zielinski and K. F. Morris, Magn. Reson. Chem., 2009, 47, 53-56.

16. J. S. Kavakka, V. Parviainen, K. Wähälä, I. Kilpeläinen and S. Heikkinen, Magn. Reson. Chem., 2010, 48, 777-781.

17. A. K. Rogerson, J. A. Aguilar, M. Nilsson and G. A. Morris, Chem. Commun., 2011, 47, 7063-7064.

18. C. Pemberton, R. Hoffman, A. Aserin and N. Garti, J. Magn. Reson., 2011, 208, 262-269.

19. A. Tal and L. Frydman, Prog. Nucl. Magn. Reson. Spectrosc., 2010, 57, 241-292. 
20

N. H. Meyer and K. Zangger, Angew. Chem. Int. Ed., 2013, 52, 7143-7146.

21. K. Kazimierczuk and V. Orekhov, Magn. Reson. Chem. 2015, 53, 921-926.

22.

K. Zangger, Prog. Nucl. Magn. Reson. Spectrosc., 2015, 8687, 1-20.

23. G. Dal Poggetto, L. Castanar, R. W. Adams, G. A. Morris and M. Nilsson, J. Am. Chem. Soc., 2019, 141, 5766-5771. A. A. Bothner-By and P. E. Johner, Biophys. J., 1978, 24, 779-790.

M. P. Williamson and D. H. Williams, J. Chem. Soc., Chem. Commun., 1981, DOI: 10.1039/C39810000165, 165-166.

26 L. A. Luck and C. R. Landis, Organometallics, 1992, 11, 1003-1005.

$27 . \quad$ C. R. Landis, L. L. Luck and J. M. Wright, J. Magn. Reson. Ser. B, 1995, 109, 44-59.

28. S. F. Lienin, R. Brüschweiler and R. R. Ernst, J. Magn Reson., 1998, 131, 184-190.

29. A. J. Simpson, G. Woods and O. Mehrzad, Anal. Chem. 2008, 80, 186-194.

30. P. Lameiras, L. Boudesocque, Z. Mouloungui, J. H. Renault J. M. Wieruszeski, G. Lippens and J. M. Nuzillard, J. Magn Reson., 2011, 212, 161-168.

31. H. Farooq, R. Soong, D. Courtier-Murias, C. Anklin and A. Simpson, Anal. Chem., 2012, 84, 6759-6766. P. Lameiras and J.-M. Nuzillard, Anal. Chem., 2016, 88, 4508-4515.

33. P. Lameiras, S. Patis, J. Jakhlal, S. Castex, P. Clivio and J. M. Nuzillard, Chem. Eur. J., 2017, 23, 4923-4928.

34. P. Lameiras, S. Mougeolle, F. Pedinielli and J.-M. Nuzillard, Faraday Discuss., 2019, 218, 233-246.

35.

E. Adair, C. Afonso, N. G. A. Bell, A. N. Davies, M. A Delsuc, R. Godfrey, R. Goodacre, J. A. Hawkes, N. Hertkorn, D. Jones, P. Lameiras, A. Le Guennec, A. Lubben, M. Nilsson, L. Paša-Tolić, J. Richards, R. P. Rodgers, C. P. Rüger, P. Schmitt-Kopplin, P. J. Schoenmakers, P. Sidebottom, D. Staerk, S. Summerfield, D. Uhrín, P. van Delft, J. J. J. van der Hooft, F. H. M. van Zelst and A. Zherebker, Faraday Discuss., 2019, 218, 247-267.

36. F. Pedinielli, J.-M. Nuzillard and P. Lameiras, Anal. Chem., 2020, 92, 5191-5199.

37. A. Seidell and W. F. Linke, Solubilities of Inorganic and Organic Compounds: A Compilation of Solubility Data from the Periodical Literature, Van Nostrand, 1952.

38. A. Smith and A. W. C. Menzies, J. Am. Chem. Soc., 1909, 31, 1183-1191.

39. X. Jiang, Y. Zhao, B. Hou, M. Zhang and Y. Bao, J. Chem Eng. Data, 2011, 56, 205-211.

40. J. M. G. Cowie and P. M. Toporowski, Can. J. Chem., 1961 39, 2240-2243.

41. M. O. Sonnati, S. Amigoni, E. P. Taffin de Givenchy, T. Darmanin, O. Choulet and F. Guittard, Green Chem., 2013 , 15, 283-306.

42. T. L. Hwang and A. J. Shaka, J. Magn. Reson. Ser. A, 1995, 112, 275-279.

43. P. Douzou and G. A. Petsko, Adv. Protein Chem. , 1984, 36, 245-361.

44. A. Gierer and K. Wirtz, Zeitschrift Für Naturforschung Section A, 1953, 8, 532-538.

45. K. Wuthrich, NMR of proteins and nucleic acids, 1986, New York: Wiley.
46.

48.

49.

D.

K. Stott, J. Stonehouse, J. Keeler, T.-L. Hwang and A. J. Shaka, J. Am. Chem. Soc., 1995, 117, 4199-4200.

K. Stott, J. Keeler, Q. N. Van and A. J. Shaka, J. Magn. Reson., 1997, 125, 302-324.

R. Brüschweiler, C. Griesinger, O. W. Sørensen and R. R. Ernst, J. Magn. Reson., 1988, 78, 178-185.

B. Plainchont, A. Martinez, S. Tisse, J. P. Bouillon, J. M. Wieruszeski, G. Lippens, D. Jeannerat and J. M. Nuzillard, J. Magn. Reson., 2010, 206, 68-73.

52.

Ē. Kupče and T. D. W. Claridge, J. Magn. Reson., 2019, 307. Spectroscopy and Spectrometry, Elsevier Ltd, 1999, DOI: 10.1016/B978-0-12-374413-5.00242-6, pp. 2204-2212.

O. Kühl, Phosphorus-31 NMR Spectroscopy: A Concise Introduction for the Synthetic Organic and Organometallic Chemist, Springer, 2009.

53. T. R. Tritton and I. M. Armitage, Nucleic Acids Res., 1978, 5, 3855-3869.

54. P. T. Robinson, T. N. Pham and D. Uhrín, J Magn Reson, 2004, 170, 97-103.

55. P. Kiraly, N. Kern, M. P. Plesniak, M. Nilsson, D. J. Procter, G. A. Morris and R. W. Adams, Angewandte Chemie International Edition, 2021, 60, 666-669. 